\title{
Stable coexistence of aerobic and anaerobic transition
}

\author{
YAMINI JANGIR ${ }^{1}$, YONGZHAO GUO ${ }^{1}$, EMILY ZAKEM ${ }^{2}$, \\ NAOMI M. LEVINE ${ }^{2}$ AND VICTORIA J. ORPHAN ${ }^{1}$ \\ ${ }^{1}$ California Institute of Technology \\ ${ }^{2}$ University of Southern California \\ Presenting Author: jangir@caltech.edu
}

In anoxic zones of the pelagic open ocean, organic matter is oxidized anaerobically, with some of it transformed into elemental forms $\left(\mathrm{N}_{2}\right.$ and the potent greenhouse gas $\left.\mathrm{N}_{2} \mathrm{O}\right)$ that are no longer bioavailable. Resource ratio theory predicts a transition from strictly aerobic metabolism in the surrounding waters to the stable coexistence of both aerobic and anaerobic metabolism [1]. Parameterizing this process using resource ratio theory can increase predictive ability of biogeochemical models. Here we are testing these model predictions under controlled conditions in the laboratory using a cultured facultative marine anaerobic heterotroph, Marinobacter sp. D2M19.

Members of the genus Marinobacter are capable of denitrification through NarGHI (membrane bound) and/or NapABC (periplasmic) protein complexes. Genes encoding for NarGHI have also been detected in the heart of oxygen minimum zones, indicating active denitrification by the community [2]. Here, we describe steady-state growth of Marinobacter sp. D2M19 under four different environmental conditions varying the available terminal electron acceptor: (1) oxygen (open system), (2) nitrate and oxygen (open system), (3) nitrate and oxygen (closed system), and (4) nitrate (closed system). Whole genome analysis of Marinobacter sp. D2M19 predicts the ability to carry out complete denitrification via NarGHI.

To test for metabolic heterogeneity within the population, assays using real time quantitative PCR and single cell resolved mRNA expression of narGHI using seqFISH were developed and used to characterize population-level and single-cell-level expression under various environmental conditions. Preliminary mRNA-FISH and RT-PCR suggests the coexistence of oxygen and nitrate reduction within the population. Our ongoing work will characterize how the substrate supply ratio affects growth dynamics and relative proportion of cells expressing narGHI in relation to model predictions. This hybrid model and laboratory study illustrates the importance of considering the potential for metabolic heterogeneity within discrete populations of microorganisms as cells dynamically adjust to maximize growth rate and cellular activities in response to a changing environment. Results will ultimately aid in improving quantitative predictions of anaerobic activity and bioavailable $\mathrm{N}$ loss at the global scale.

[1] Zakem, Mahadevan, Lauderdale, \& Follows (2020), ISME Journal, 14(1), 288-301.

[2] Ulloa, Canfield, DeLong, Letelier, \& Stewart (2012). PNAS, 109(40), 15996-16003. 